\title{
Functional Magnetic Resonance Imaging Responses Relate to Differences in Real-World Social Experience
}

\author{
Naomi I. Eisenberger, Shelly L. Gable, and Matthew D. Lieberman \\ University of California, Los Angeles
}

\begin{abstract}
Although neuroimaging techniques have proven powerful in assessing neural responses, little is known about whether scanner-based neural activity relates to real-world psychological experience. A joint functional magnetic resonance imaging (fMRI)/experience-sampling study investigated whether individual differences in neurocognitive reactivity to scanner-based social rejection related to: (a) moment-tomoment feelings of social rejection during real-world social interactions ("momentary social distress") and (b) the extent to which these momentary feelings corresponded with end-of-day global assessments of social disconnection ("end-of-day social disconnection"). Individuals who showed greater activity in regions associated with affective and pain processing (dorsal anterior cingulate cortex, amygdala, periaqueductal gray) during scanner-based social rejection reported feeling greater momentary social distress during their daily social interactions. In contrast, individuals who showed greater activity in regions associated with memory and self-referential memory encoding (hippocampus, medial prefrontal cortex) showed a stronger correspondence between momentary social distress and end-of-day social disconnection, such that greater momentary social distress was associated with greater end-of-day social disconnection. These findings complement previous work showing a dissociation between momentary and retrospective reports of affect and suggest that these processes rely on dissociable neural systems.
\end{abstract}

Keywords: fMRI, experience-sampling, social rejection, neural, real-world social experience, momentary versus retrospective reports of affect

In the past decade, there has been a surge in the number of studies that have used neuroimaging techniques to elucidate the neural correlates of psychological experience. Despite the fact that the goal of this work is presumably to understand how the human mind functions in everyday life, relatively little is known about how neural activity during scanner-based tasks relates to realworld experience. Although it may be reasonable to assume that neural activity during certain, basic psychological processes generalizes from the functional magnetic resonance imaging (fMRI) scanner to the real world, it is less clear whether these same assumptions can be made about the processing of social and emotional information. The real-world instantiation of these processes, embedded in complex social dynamics and ongoing social relationships, bears little resemblance to the performance of simple tasks in the confined and controlled context of an fMRI experiment. With the increasing interest in the neural underpinnings of social and emotional processes, it is important to assess whether neural activity to specific social or emotional experiences in the scanner has meaningful correlates in the real world.

Naomi I. Eisenberger, Shelly L. Gable, and Matthew D. Lieberman, Department of Psychology, Franz Hall, University of California, Los Angeles.

This research was funded by a postdoctoral research fellowship from the National Institutes of Mental Health to N. I. Eisenberger (T32 MH-019925) and by grants from the National Institutes of Mental Health to M. D. Lieberman (R21MH66709-01; R21MH071521-01). We thank the staff of the UCLA Brain Mapping Center for their assistance.

Correspondence concerning this article should be addressed to Naomi I. Eisenberger, Department of Psychology, 300 Medical Plaza, University of California, Los Angeles 90095-7076. E-mail: neisenbe@ucla.edu
Previous work has shown that neural activation during an episode of social rejection in the scanner is strongly correlated with self-reports of social distress taken immediately after the rejection episode (e.g., "I felt rejected," "I felt invisible"). Individuals who showed greater activity in the dorsal anterior cingulate cortex (dACC) during a social rejection episode reported feeling more distressed by the rejection episode ( $r=0.88$; Eisenberger, Lieberman, \& Williams, 2003). However, it is not clear whether neural responses to an experimental episode of social rejection relate to real-world social experience. In other words, does dACC activity in response to an experimental episode of social rejection in the scanner relate to an individual's tendency to feel socially rejected or accepted during real-world social interactions? Moreover, does dACC activity in response to an experimental episode of social rejection relate to the extent to which momentary feelings of social rejection or acceptance are incorporated into more global beliefs about one's social standing? Because it is not yet possible to directly assess whole-brain neural activity during naturalistic, real-world social encounters, the present study investigated whether neural responses during an experimental episode of social rejection within the fMRI scanner correlated with real-world experiences during ongoing social interactions.

The first goal of the present study was to examine whether neural activity in response to social rejection in the scanner related to moment-to-moment feelings of social rejection in daily social interactions. To investigate neural activity in response to social rejection, participants were scanned while they were excluded during a virtual ball-tossing game, allegedly with two other individuals (as has been done previously; Eisenberger et al., 2003). To assess moment-to-moment feelings of social distress, participants 
completed a 10-day experience-sampling study in which they were randomly signaled at different times during the day and asked to report on their feelings of social distress in their most recent social interaction ("momentary social distress;" e.g., "I felt accepted/ rejected by my interaction partner"). We hypothesized that individuals who showed greater dACC activity in response to an experimental episode of social rejection in the scanner would also report feeling higher levels of momentary social distress during their daily social interactions. In other words, individuals who are more sensitive to scanner-based social rejection, as indexed by greater dACC activity, should also be more sensitive to rejectionrelated cues or the possibility of rejection in their daily social interactions (even if these interactions do not involve explicit rejection like that seen in the social exclusion task) and thus report greater momentary social distress.

A second goal of the present study was to investigate whether neural activity in response to scanner-based social rejection related to the extent to which momentary social distress during daily social interactions corresponded with end-of-day global assessments of social disconnection. Previous work has shown that real-time experience and retrospective reports of that experience do not necessarily correspond (Fredrickson \& Kahneman, 1993; Kahneman, Fredrickson, Schreiber, \& Redelmeier, 1993; Redelmeier \& Kahneman, 1996; Updegraff, Gable, \& Taylor, 2004). For example, two individuals may experience similar levels of positive and negative affect over the course of a day, but then retrospectively report different levels of well-being at the end of the day, depending on which experiences are retrieved when making that reflective assessment. Accordingly, a recent study demonstrated that individuals who scored higher in trait-level approach motivation, compared with those who scored lower, were found to give greater weight to momentary positive experiences when making end-ofday judgments of life satisfaction (Updegraff et al., 2004). In other words, for those higher in trait approach motivation, there was a stronger correspondence between momentary positive affect and end-of-day life satisfaction.

In the present study, we investigated whether neural responses to scanner-based social rejection provided a meaningful index for how momentary feelings of social distress during daily social interactions corresponded with end-of-day assessments of social disconnection. To examine this, participants provided a global assessment of social disconnection at the end of each of the 10 days ("end-of-day social disconnection;" e.g., "Today, I generally felt accepted by others: strongly agree/strongly disagree"), and correlations were computed between momentary social distress and end-of-day social disconnection ratings across the 10-day period. This correlation provided an index of the extent to which momentary social distress corresponded with end-of-day assessments of social disconnection. We then investigated how neural activity during social rejection in the scanner related to this correspondence measure. This analysis allowed us to examine whether certain kinds of neural activity during an episode of rejection increased the likelihood of momentary social distress corresponding with end-of-day reports. ${ }^{1}$

If a strong correspondence between momentary social distress and end-of-day social disconnection is a function of memory encoding processes (such that individuals who encode these experiences more deeply are more likely to show end-of-day reports that correspond more closely with momentary experiences), we might expect that individuals who show greater activity in the hippocampus and other medial temporal lobe (MTL) regions, shown to be associated with memory encoding processes (Brewer, Zhao, Desmond, Glover, \& Gabrieli, 1998; Wagner et al., 1998), during social rejection would show a stronger correspondence between momentary social distress and end-of-day social disconnection. Greater activity in these regions during real-world experiences of social rejection would increase the likelihood that these experiences are encoded into long-term memory and then more easily retrieved later on when asked to reflect on global feelings of social disconnection.

Finally, we examined how self-reported trait measures, known to predict feelings of social distress (rejection sensitivity, social anxiety, neuroticism), related to real-world social experience as well. These measures were included to examine how neural assessments compared with self-report assessments in relating to both momentary social distress and the correspondence between momentary social distress and end-of-day social disconnection.

\section{Method}

\section{Participants and Design Overview}

Forty-two healthy participants (22 female; mean age $=21.12$ years, $S D=3.94$ ), all right-handed, provided written informed consent to participate in this study. Experimental procedures were approved by the Human Subjects Protection Committee at the University of California, Los Angeles.

Participants came in at two different times to complete the study. At Time 1, participants completed self-report measures related to the tendency to experience social distress (neuroticism, social anxiety, rejection sensitivity) and then began the 10-day experience-sampling study in which they were randomly signaled at different times during the day to report on their most recent social interaction. At Time 2 (which took place 1 to 2 weeks following the completion of the experience-sampling study), participants completed a neuroimaging component in which they were socially excluded in the fMRI scanner.

The daily experience-sampling procedure was conducted prior to the neuroimaging component to ensure that participants had a reasonable number of daily entries prior to being scanned. Since each neuroimaging session is quite expensive, only participants who had enough daily entries to allow for the examination of how these daily assessments related to neural activity participated in the scanning phase of the study. Of the 42 participants who began the daily experience study, 33 completed the neuroimaging component (20 females). Reasons for not completing the neuroimaging component included: not completing the experience-sampling compo-

\footnotetext{
${ }^{1}$ Both of our main analyses focused on momentary social distress in daily life, either: (a) on its own or (b) as it corresponds with end-of-day self-reports of social disconnection. We focused on these assessments because neural responses to social rejection in the scanner are most likely to serve as a proxy for momentary experiences in social interactions. We did not examine the direct relation between neural responses to scannerbased social rejection and end-of-day self-reports because social rejection in the scanner is not a meaningful proxy for these retrospective judgments, and the rejection experience in the scanner is not the target of these retrospective judgments.
} 
nent $(n=2)$, not having enough entries to be considered for scanning $(n=5)$, data loss $(n=1)$, and claustrophobia $(n=1)$. In addition, three participants were excluded from the neuroimaging analyses: one due to excessive motion, one due to being an outlier on neural data (greater than 3 SDs below the mean for the sample), and one due to prior experience with the social exclusion task. The final sample consisted of 30 healthy participants (18 women; mean age $=20.73, S D=3.23$ ).

\section{Individual Difference Measures}

Before completing the daily experience-sampling assessment, participants completed several measures assessing traits known to predict feelings of social distress during social interactions. Specifically, participants completed measures of rejection sensitivity (e.g., "I sometimes take criticism too hard;" Mehrabian, 1976), social anxiety (e.g., "I feel anxious when I speak in front of a group;" Fenigstein, Scheier, \& Buss, 1975), and neuroticism (e.g., "Are your feelings easily hurt?" "Would you call yourself a nervous person?" Eysenck \& Eysenck, 1975). The alpha reliabilities of these scales were $0.56,0.76$, and 0.88 , respectively. These measures were assessed to determine how neural assessments compared to self-reported trait measures in relating to social experiences.

\section{Daily Experience Assessment}

To assess momentary social distress during daily social interactions, participants were loaned a PalmPilot device running the Experience Sampling Program (Barrett \& Barrett, 2001), which administered the relevant questions. Over the course of 10 days, participants were randomly signaled at different times during the day and, once signaled, answered questions on the PalmPilot related to their most recent social interaction. Social interactions were defined as any interaction with one or more individuals that lasted for 5 minutes or longer, but not including email or webbased interactions.

Participants were given a limited amount of time to respond to each signal, and if they did not respond during that time, the PalmPilot shut off. If participants responded to a signal within the time window, they were first asked whether they were able to complete an interaction entry; if the participant answered no, the PalmPilot shut off. If they answered yes, they were then asked if they had had a new interaction since the last signal. If the participant answered no, the questionnaire ended; if the participant answered yes, the participant completed the questionnaire and could not return to previously answered questions. Again, if participants did not respond to a question item within a certain time window, the PalmPilot shut off, preventing participants from returning to a questionnaire at a later point in time.

Participants were informed that they could turn the volume off on the PalmPilot when they could not be disturbed, such as while driving, during class, or in important meetings. In addition, the PalmPilot was set so that it would only signal participants during hours when they reported they would typically be awake. Because participants were told that they could turn off the volume on the PalmPilot when they could not be disturbed, participants were signaled more frequently to ensure that enough assessments were collected when the PalmPilot's volume was on. In order to obtain approximately four daily assessments, the PalmPilots were set to go off at eight different times during the day (regardless of when the volume was on or off). Similar techniques have been validated and used widely (Reis \& Gable, 2002).

To assess momentary social distress, participants rated how socially disconnected or rejected they felt during their most recent social interaction using two scale items: "I felt connected to/distant from my interaction partner" and "I felt accepted/rejected by my interaction partner" (modified from the Need-Threat scale; Williams, Cheung, \& Choi, 2000). These items were rated on a scale ranging from (1) very accepted (or connected to) to (7) very rejected (or distant from). To compute momentary social distress, average scores of these two items were computed for each subject across each day. The reliability of this measure was strong $(\alpha=0.83)$.

At the end of each day, participants provided end-of-day retrospective reports of social disconnection through a brief questionnaire that was emailed to them each evening. To assess end-of-day feelings of social disconnection, participants rated the extent to which they agreed with two statements: "Today, I generally felt connected to others" and "Today, I generally felt accepted by others" (modified from the Need-Threat scale; Williams et al., 2000). These items were rated on a scale ranging from (1) strongly agree to (7) strongly disagree. ${ }^{2}$ The reliability of this measure was strong $(\alpha=0.85)$.

The extent to which momentary social distress correlated with end-of-day assessments of social disconnection was used as an index of the degree to which these social experiences were integrated into global assessments of social disconnection. Thus, individuals with a large, positive correlation coefficient had a stronger correspondence between momentary and end-of-day measures, such that higher levels of social distress during the day were associated with higher levels of social disconnection at the end of each day. Individuals with a small correlation coefficient (either positive or negative) showed little correspondence between momentary and end-of-day measures. Finally, individuals with a large, negative correlation coefficient showed a correspondence between momentary and end-of-day measures in the unexpected direction, such that higher levels of social distress during the day were associated with lower levels of self-reported social disconnection at the end of the day. The average correlation, for each participant, between momentary social distress and end-of-day social disconnection across the 10-day assessment period was medium-sized (average $r=0.31$; Rosenthal \& Rosnow, 1991).

\section{Cyberball Social Exclusion Task}

To assess neurocognitive reactivity to social rejection, participants were scanned while completing the Cyberball social exclusion task, in a manner similar to previous work (Eisenberger et al., 2003; Williams et al., 2000). Participants were told that they would be playing a virtual ball-tossing game with two other individuals who were also in fMRI scanners. In reality, however, there were no other players; participants were playing with a preset computer program. Each game began with a still picture of the two virtual players in the upper corners

\footnotetext{
${ }^{2}$ We refer to this scale as a measure of social disconnection rather than a measure of social connection only for ease of interpretation, so that both momentary social distress and end-of-day social disconnection are similarly valenced. Ideally, future investigations would use items worded in the same way for both the momentary and end-of-day reports.
} 
of the screen and a hand, representing the participant, in the lowercenter portion of the screen. The participant's name was displayed below the hand while two other names were displayed below each of the two virtual players' animated cartoon representations. After 9 seconds, the cartoon player in the upper left-hand corner started the game by throwing the ball to either the other cartoon player or the participant. The participant could return the ball to one of the players by pressing one of two keys on a button box. The Cyberball program was set for 60 throws per game, with the computer players waiting 0.5 to 3.0 seconds (determined randomly) before making a throw to heighten the sense that the participant was actually playing with other individuals.

During the task, participants completed two scans. In the first scan (inclusion), participants played with the two other players for the entire scanning period, with each virtual player throwing the ball to the participant on approximately $50 \%$ of the throws. In the second scan (exclusion), participants only received the ball for a total of seven throws and were then excluded for the rest of the scan when the two players stopped throwing the ball to the participant (60-90 seconds). ${ }^{3}$ Immediately following the scanning session, participants completed a measure of self-reported social distress, in which they were asked to rate how socially distressed they felt during the final ball-tossing game (e.g., "I felt rejected," "I felt invisible;" Williams et al., 2000).

\section{fMRI Data Acquisition and Data Analysis}

Data were acquired on a Siemens Allegra 3T scanner. Head movements were restrained with foam padding and surgical tape placed across each participant's forehead. For each participant, a highresolution structural T2-weighted echo-planar imaging volume (spinecho; time for repetition (TR) $=5000 \mathrm{~ms}$; time for echo $(\mathrm{TE})=33$ ms; matrix size $128 \times 128 ; 36$ axial slices; field of view $(\mathrm{FOV})=20$ $\mathrm{cm}$; 3-mm thick, skip 1-mm) was acquired coplanar with the functional scans. Two functional scans were acquired (echo planar T2*weighted gradient-echo, $\mathrm{TR}=3000 \mathrm{~ms}, \mathrm{TE}=25 \mathrm{~ms}$, flip angle $=$ $90^{\circ}$, matrix size $64 \times 64,36$ axial slices, FOV $=20$-cm; 3 -mm thick, skip 1-mm), each lasting 2 minutes and 30 seconds.

The imaging data were analyzed using statistical parametric mapping (SPM'99; Wellcome Department of Cognitive Neurology, Institute of Neurology, London, United Kingdom). Images for each participant were realigned to correct for head motion, normalized into a standard stereotactic space as defined by the Montreal Neurological Institute, and smoothed with an $8 \mathrm{~mm}$ Gaussian kernel, full width at half maximum. For each participant, periods of inclusion and exclusion were modeled as epochs based on the length of that participant's inclusion and exclusion episodes, which were individually timed for each participant (these varied slightly between participants due to the random delay assigned to the virtual players when throwing the ball). After the task was modeled for each participant, planned comparisons were computed as linear contrasts to investigate neural activity during the exclusion compared to the inclusion episode.

To assess correlations between momentary social distress and neural activity, the measure of momentary social distress was entered as a regressor into a random effects, whole-brain group analysis, comparing activations for the exclusion episode to activations for the inclusion episode. Similarly, to assess how neural reactivity moderated the relationship between momentary social distress and end-of-day social disconnection, correlations between momentary social distress and end-of-day social disconnection across the 10-day period were calculated for each participant. Two participants were excluded from this analysis because they did not have enough momentary social interaction entries and end-of-day entries completed on the same days to compute a correlation score. The correlations between momentary social distress and end-ofday social disconnection were then entered separately as regressors into a random effects, whole-brain group analysis, comparing activations for the exclusion compared to the inclusion episode. All analyses were thresholded using an uncorrected $p$ value of .005 combined with a cluster size threshold of 10 voxels (Forman et al., 1995). All coordinates are reported in Montreal Neurological Institute (MNI) format.

\section{Results}

\section{Compliance}

Participants responded to an average of four $(S D=1.24)$ signals per day. Of these four signals, participants reported that they could not complete approximately one of the signals $(M=0.7 ; S D=$ $0.76)$ and that they had had no new interaction on approximately one signal $(M=0.7 ; S D=0.69)$. On average, they completed approximately 2.5 assessments of momentary social distress per day $(S D=1.21)$. Neither overall compliance (responding to the PalmPilot device) nor the number of momentary social distress ratings made per day was significantly associated with selfreported levels of momentary social distress or with any of the self-report measures that might relate to social distress.

With regard to the end-of-day assessments, participants completed an average of 9.6 end-of-day entries $(S D=1.10)$ across the 10-day period. Compliance in completing the end-of-day assessments was also not associated with end-of-day reports of social disconnection or with any of the self-report measures that might relate to social distress.

\section{Does Neural Activity During Scanner-Based Social Rejection Task Relate to Momentary Social Distress in Real-World Social Interactions?}

We first examined whether neural activity during the exclusion episode, relative to the inclusion episode, correlated with momentary social distress assessed during daily social interactions across the 10-day assessment period. To do this, we regressed average social distress scores across the 10-day period into a whole-brain group analysis $(p<.005$, 10-voxel extent threshold). Results revealed that, in response to the scanner-based social rejection episode, individuals who showed greater activity in the left dACC reported greater levels of momentary social distress during their social interactions $(-12,32,38 ; r=0.53, p<.005$; see Figure 1A). Moreover,

\footnotetext{
${ }^{3}$ Although it would have been ideal to counterbalance the order of the inclusion and exclusion scans across participants, having the exclusion scan come before the inclusion scan would likely change the meaning of the inclusion scan for participants. Thus, participants who were first excluded might subsequently worry about being excluded again or anticipate that another exclusion episode was possible.
} 


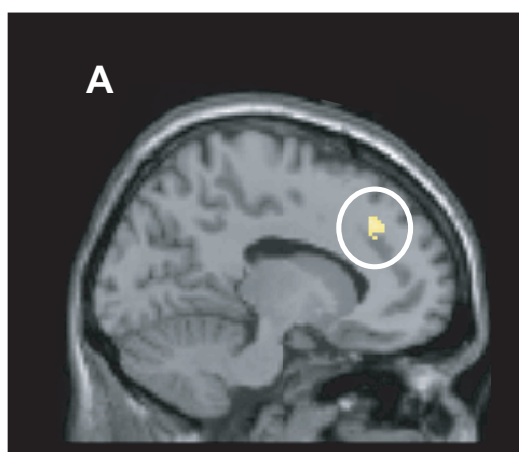

dACC

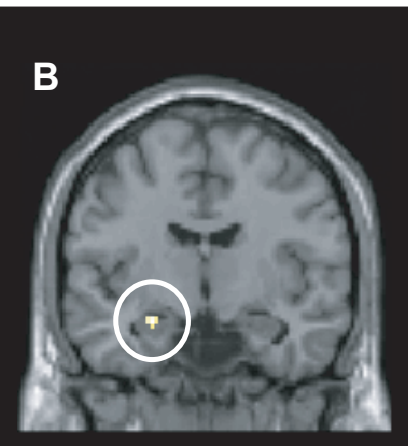

Amygdala

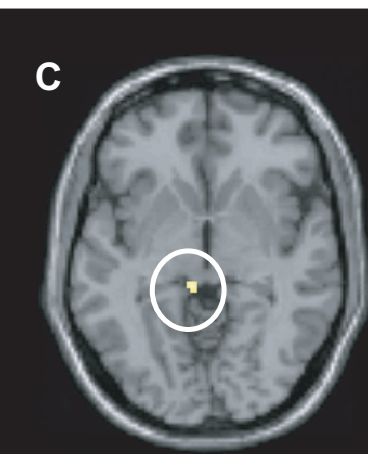

PAG
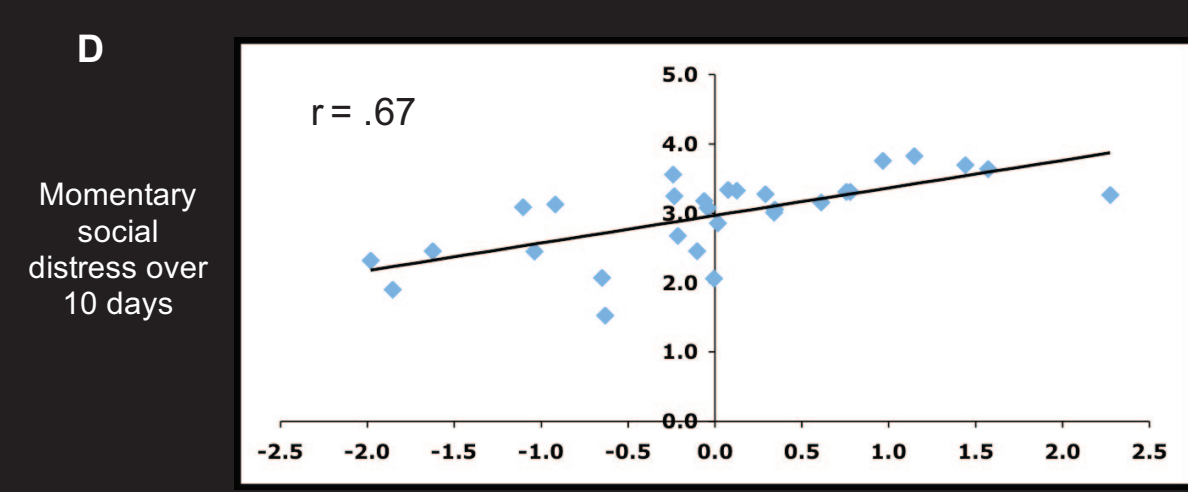

Predicted values for combined dACC/amygdala/PAG activity

Figure 1. Neural activity during exclusion, relative to inclusion, that correlated positively with momentary social distress during daily social interactions in the (A) dorsal anterior cingulate cortex (dACC), (B) amygdala, and (C) periaqueductal gray (PAG). (D) Scatterplot showing the regression of dACC, amygdala, and PAG activity onto momentary social distress scores. The $\mathrm{x}$-axis represents the predicted values from including $\mathrm{dACC}$, amygdala, and PAG activity in a regression analysis estimating momentary social distress. The predicted values are an average of the activity in these three regions weighted according to the weights in the regression equation. The $y$-axis represents average momentary social distress scores across the 10-day assessment period. Each point represents the data from a single participant.

greater activity in this region of the dACC was significantly associated with greater self-reported social distress in response to the Cyberball game $(r=0.43, p<.05)$. We also found that individuals who showed greater activity in the left amygdala and left periaqueductal gray (PAG), regions associated with affective and pain processing (Davis \& Whalen, 2001; Peyron, Laurent, \& Garcia-Larrea, 2000; Rainville, Duncan, Price, Carrier, \& Bushnell, 1997), also reported significantly greater levels of momentary social distress during their social interactions $(r=0.57$ and 0.55 , respectively; see Figure $1, \mathrm{~B}$ and $\mathrm{C}$; see Table 1a for a complete list of activations). ${ }^{4}$ These two regions, however, were not significantly correlated with selfreported social distress in response to the Cyberball game, consistent with our previous findings (Eisenberger et al., 2003). There were no significant negative correlations between neural activity and momentary social distress.

We also assessed several traits known to predict feelings of social distress during social interactions (rejection sensitivity, social anxiety, neuroticism) to determine how these selfreported trait measures compared with the neural assessments in relating to real-world social experiences. Together, the neural activity in the AACC, amygdala, and PAG during a single social rejection episode in the fMRI scanner accounted for $45 \%$ of the between-subjects variance in momentary social distress, averaged across the 10-day period, $F(3,28)=6.90, p<.005$; see Figure 1D. In contrast, self-reported rejection sensitivity, social anxiety, and neuroticism together accounted for $28 \%$ of the variance in momentary social distress, $F(3,31)=3.53, p<.05$. Moreover, activity in the dACC, amygdala, and PAG was still significantly associated with momentary social distress after controlling for these three self-report measures, $F(3,22)=$ $5.84, p<.005$; however, the self-report measures were no longer significantly associated with momentary social distress after controlling for the neural measures, $F(3,22)=1.97, p=$ $.15, n s$. Thus, neural activity in response to a single social

\footnotetext{
${ }^{4}$ The PAG activation should be interpreted with caution due to the small size of this brainstem nucleus and the lack of spatial resolution afforded by fMRI to accurately identify neural regions of this size.
} 
Table 1

Neural Activity Related to (A) Momentary Social Distress and (B) the Correspondence Between Momentary Social Distress and Endof-Day Social Disconnection

\begin{tabular}{|c|c|c|c|c|c|c|}
\hline Region & $\begin{array}{l}\text { Brodmann's } \\
\text { Area (BA) }\end{array}$ & \multicolumn{3}{|c|}{$\begin{array}{c}\text { Montreal Neurological Institute } \\
\text { Coordinate }\end{array}$} & \multirow[t]{2}{*}{$k$} & \multirow[t]{2}{*}{$\begin{array}{l}\text { Correlation } \\
\qquad(r)\end{array}$} \\
\hline \multicolumn{5}{|c|}{$\begin{array}{l}\text { (A) Correlations between neural activity and } \\
\text { momentary social distress during } \\
\text { 10-day assessment period }\end{array}$} & & \\
\hline $\mathrm{dACC}$ & 32 & -12 & 32 & 38 & 45 & 0.53 \\
\hline Amygdala & & -24 & -8 & -18 & 14 & 0.57 \\
\hline PAG & & -6 & -32 & -4 & 16 & 0.55 \\
\hline Caudate & & -16 & 16 & 14 & 37 & 0.54 \\
\hline Medial parietal cortex & 7 & -12 & -60 & 68 & 14 & 0.54 \\
\hline Cerebellum & & 26 & -54 & -38 & 18 & 0.57 \\
\hline & & 0 & -60 & -30 & 10 & 0.52 \\
\hline Brainstem & & -6 & -20 & -20 & 39 & 0.58 \\
\hline & & 6 & -16 & -26 & 36 & 0.56 \\
\hline \multicolumn{7}{|c|}{$\begin{array}{l}\text { (B) Correlations between neural activity and } \\
\text { the correspondence between momentary } \\
\text { social distress and end-of-day social } \\
\text { disconnection }\end{array}$} \\
\hline Hippocampus & & -30 & -8 & -18 & 21 & 0.54 \\
\hline Medial prefrontal cortex & 10 & -14 & 60 & 10 & 16 & 0.53 \\
\hline Temporal cortex & $21 / 38$ & 46 & 10 & -26 & 15 & 0.54 \\
\hline \multirow[t]{2}{*}{ Fusiform gyrus } & 37 & 36 & -56 & -8 & 259 & 0.68 \\
\hline & 20 & -46 & -22 & -22 & 10 & 0.53 \\
\hline Caudate & & -16 & 14 & 2 & 12 & 0.53 \\
\hline Corpus callosum & & -2 & -34 & 20 & 50 & 0.60 \\
\hline \multirow[t]{4}{*}{ Cerebellum } & & 28 & -66 & -26 & 175 & 0.64 \\
\hline & & -22 & -84 & -36 & 52 & 0.60 \\
\hline & & 40 & -54 & -20 & 50 & 0.59 \\
\hline & & -22 & -64 & -14 & 11 & 0.53 \\
\hline
\end{tabular}

Note. $\quad \mathrm{dACC}=$ dorsal anterior cingulate cortex; PAG = periaqueductal gray. Neural activity is always taken from the contrast of exclusion minus inclusion conditions during the Cyberball game. All coordinates are in MNI coordinate space. Significance was determined using $p<.005$ with a 10 -voxel extent threshold.

rejection episode in the scanner provided a strong index of the tendency to experience momentary social distress in daily life.

\section{Does Neural Activity During Scanner-Based Social Rejection Task Relate to the Extent to Which Momentary Social Distress Corresponded With End-of-Day Assessments of Social Disconnection?}

We also examined which neural regions were involved in the extent to which momentary social distress during daily social interactions corresponded with end-of-day assessments of social disconnection across the 10-day assessment period. To create an index of the extent to which momentary social distress corresponded with end-of-day social disconnection, we computed a correlation, for each subject, between momentary social distress and end-of-day social disconnection across the 10-day assessment period. The resulting correlation coefficients $(M=0.30 ; S D=$ 0.37 ) ranged from $r=.92$ (greater momentary social distress was associated with greater end-of-day social disconnection) to $r=$ -0.55 (greater momentary social distress was associated with lower end-of-day social disconnection). After computing the correlation coefficient for each subject, we then regressed these correlation coefficients into a whole-brain group analysis $(p<.005$, 10-voxel extent threshold).
Activity in the dACC, amygdala, and PAG in response to experimental social rejection did not significantly relate to the correspondence between momentary social distress and end-of-day social disconnection in whole-brain analyses (see Table 2 for intercorrelations among study variables); however, activity in the left hippocampus and medial prefrontal cortex (MPFC) did (see Figure 2, A and B; see Table 1b for a complete list of activations). In response to an experimental episode of social rejection, individuals who produced greater activity in the hippocampus, a neural region that has been shown to be associated with episodic memory encoding (Brewer et al., 1998; Wagner et al., 1998), and in the MPFC, a neural region that has been shown to be associated with self-referential or autobiographical memory encoding (Cabeza et al., 2004; Macrae, Moran, Heatherton, Banfield, \& Kelley, 2004) among other functions (Gallagher \& Frith, 2003; Gusnard, Akbudak, Shulman, \& Raichle, 2001; Wager, Phan, Liberzon, \& Taylor, 2003), evidenced a stronger correspondence between momentary social distress and end-of-day reports of social disconnection, such that greater momentary social distress during the day was associated with greater end-of-day assessments of social disconnection. Neither of these activations was significantly correlated with self-reported social distress in response to the Cyberball game. These findings suggest that those who demonstrate a strong correspondence between momentary and end-of-day measures 
Table 2

Correlations Between the Daily Experience Assessments and Neural Activity in Response to the Cyberball Game

\begin{tabular}{lcc}
\hline & $\begin{array}{c}\text { Momentary } \\
\text { social distress }\end{array}$ & $\begin{array}{c}\text { Correspondence }^{\text {measure }} \\
\text { merrespondence measure }_{\text {Cor }}\end{array}$ \\
\hline dACC $(-12,32,38)$ & 0.28 & \\
Amygdala $(-24,-8,-18)$ & $0.53^{* * *}$ & 0.14 \\
PAG $(-6,-32,-4)$ & $0.57^{* * *}$ & 0.20 \\
Hippocampus $(-30,-8,-18)$ & $0.55^{* * *}$ & 0.07 \\
MPFC $(-14,60,10)$ & 0.30 & $0.54^{* * * *}$ \\
\hline
\end{tabular}

Note. $\mathrm{dACC}=$ dorsal anterior cingulate cortex $; \mathrm{PAG}=$ periaqueductal gray; $\mathrm{MPFC}=$ medial prefrontal cortex.

${ }^{a}$ Correspondence measure refers to the correlation between momentary social distress and end-of-day social disconnection for each participant. A positive correspondence measure indicates that greater social distress is associated with greater end-of-day social disconnection.

${ }^{* * *} p<.005 ;$ all others, $p>.12$

may show greater activity in neural regions associated with memory and self-referential memory encoding processes, and thus may encode these events more deeply, making them easier to retrieve when making end-of-day global assessments of social disconnection.

In addition, whereas hippocampal and MPFC activity together accounted for $37 \%$ of the variance in the relationship between momentary social distress and end-of-day social disconnection, $F(2,26)=6.89, p<.005$; Figure $2 \mathrm{C}$, the self-reported trait measures (rejection sensitivity, neuroticism, and social anxiety) accounted for only $12 \%$ of the variance in this relationship, which was not a statistically significant amount, $F(3,28)=1.10, p=.37$, $n s$. Moreover, when controlling for the three self-report measures, hippocampal and MPFC activity was still significantly associated with the correspondence between momentary social distress and end-of-day social disconnection, $F(2,21)=4.63, p<.05$.

\section{Discussion}

The present study found that neural activity assessed within the fMRI scanner has a meaningful relationship with real-world social and emotional experience. Activity in separate neural systems in response to an experimental episode of social rejection in the scanner provided a meaningful index for how an individual experiences social relationships in daily life as well as the extent to which an individual may encode those experiences into more global assessments of social disconnection.

We found that individuals who generated greater dACC, amygdala, and PAG responses to a single episode of social rejection in the scanner also reported experiencing greater levels of momentary social distress in their daily social interactions. This is a notable finding given that this neural activity was assessed during a single episode of social rejection that is probably quite unlike what most individuals experience in their daily lives (most real-world experiences involving social rejection are likely to be more subtle than what occurs during Cyberball). However, the strong correlation between neural responses to social rejection and self-reports of social distress during real-world interactions suggests a core sensitivity to experiences of social connection or rejection, such that those who are the most sensitive to an experimental episode of social rejection in the scanner are also most sensitive to these types of experiences in their everyday lives. It should be noted, however, that it is not yet clear why certain neural regions (e.g., amygdala, PAG) that related to real-world experiences of social distress did not relate to self-reported social distress following the Cyberball task itself, whereas other neural regions (e.g., dACC) related to both. Future work will be needed to examine the role that these regions play in the specific types of feelings or cognitions that occur in response to the Cyberball game.

We also found that individuals who showed greater hippocampal and MPFC activity during an experimental episode of social rejection demonstrated a greater correspondence between momentary social distress and end-of-day social disconnection. Notably, the neural regions associated with a greater correspondence between momentary social distress and retrospective reports of social disconnection are similar to those found in neuroimaging studies of memory encoding (hippocampus: Brewer et al., 1998; Wagner et al., 1998) as well as neuroimaging studies of self-referential processing (MPFC: Gusnard et al., 2001), including self-referential and autobiographical memory encoding (MPFC: Cabeza et al., 2004; Macrae et al., 2004). In these memory-encoding studies, individuals who demonstrated greater activity in the hippocampus when viewing presented stimuli or in the MPFC when viewing self-referential stimuli were more likely to remember those stimuli in a subsequent memory test. In a similar fashion, the present data suggests that social experiences that are more deeply encoded when they occur may then be more easily retrieved when making global assessments of social disconnection at the end of the day.

These findings have several important implications. First, they demonstrate that certain types of scanner-based neural activity have meaningful correlates in real-world experience. Second, they point to a double dissociation in the neural systems associated with momentary and retrospective reports of social disconnection (Lieberman, 2007). As revealed here, the brain regions associated with momentary social distress (dACC, amygdala, PAG) were not significantly associated with the correspondence between momentary social distress and end-of-day assessments of social disconnection, and the brain regions associated with this correspondence measure (MPFC, hippocampus) were not significantly associated with momentary social distress (see Table 2). These findings map nicely onto previous behavioral work showing that moment-tomoment and retrospective reports of affect do not necessarily correspond (Fredrickson \& Kahneman, 1993; Kahneman et al., 1993; Redelmeier \& Kahneman, 1996; Updegraff et al., 2004) and suggest that part of the reason for this may be due to the fact that these processes rely on the computational substrates of two separate neural systems.

This dissociation may shed new light on ways to investigate altered emotional experience in certain clinical populations. For example, it has been shown that patients with amygdala damage show no differences from normal controls in the magnitude or frequency of self-reported positive or negative affect (Anderson \& Phelps, 2002), suggesting that the amygdala may not be necessary for the generation of affective experience. However, in this study, reports of affect were assessed at the end of the day in a retrospective manner ("surveying the feelings and 


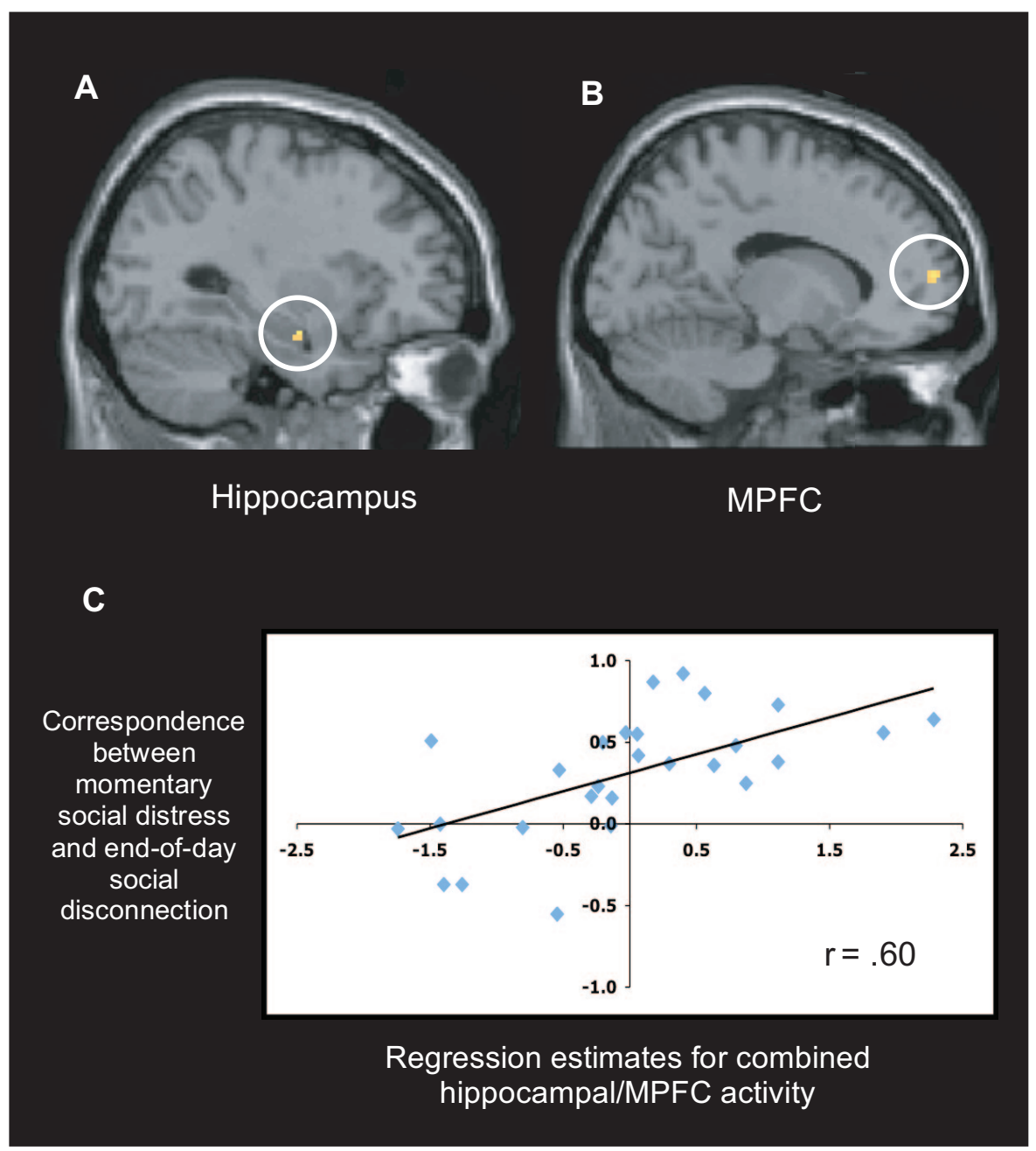

Figure 2. Neural activity during exclusion, relative to inclusion, that correlated positively with the correspondence between momentary social distress and end-of-day social disconnection in the (A) hippocampus and (B) medial prefrontal cortex (MPFC). (C) Scatterplot showing the regression of hippocampal and MPFC activity onto correspondence scores between momentary social distress and end-of-day social disconnection. The x-axis represents the predicted values from including hippocampal and MPFC activity in a regression analysis estimating the correspondence between momentary social distress and end-of-day social disconnection. The predicted values are an average of the activity in these two regions weighted according to the weights in the regression equation. The y-axis represents the correspondence between momentary social distress and end-ofday social disconnection. Positive correlations represent a higher correspondence between momentary and end-of-day measures, such that greater feelings of social distress during the day are associated with greater reports of social disconnection at the end of the day. Each point represents the data from a single participant.

emotions that they had experienced over the course of that day") and thus may reflect spared memory encoding processes rather than intact affective experience. Thus, it is possible that amygdala-damaged patients could still have altered moment-tomoment experiences of affect, but show normal retrospective reports of affect. To determine whether the amygdala is involved in moment-to-moment affective experience, it is necessary to see if amygdala-damaged patients report moment-tomoment experiences of affect (rather than retrospective experiences of affect) that are different from those of healthy controls.
More generally, this functional dissociation may have important implications for understanding how neural reactivity to certain tasks predicts future behaviors and decisions. Because decisions are often based on memories of what was liked or disliked, decisions may be more dependent on what has been encoded into memory and integrated into global self-views rather than what was actually experienced in the moment (Morewedge, Gilbert, \& Wilson, 2005). Thus, even though certain neural regions, such as the $\mathrm{dACC}$, may be critical determinants of momentary experiences of distress during a rejection episode, activity in these regions may not exclusively 
determine how an individual remembers the rejection episode, integrates it into self-views, and then uses that information to make future decisions. Rather, hippocampal and MPFC activity during a rejection episode may relate more strongly to the extent to which an experience is encoded and then later retrieved for making judgments or decisions. Thus, two people could have similarly strong reactions to social rejection while it is occurring, but the individual who encodes this experience into memory may be more likely to retrieve this memory and use it when making reflective social or self-judgments or when making explicit decisions regarding future social encounters.

This study also has a number of limitations. First, although one of the main goals of the study was to investigate whether neural responses to scanner-based social rejection related to real-world feelings of rejection, the task used to assess neural responses to social rejection (Cyberball) is most likely not all that similar to what individuals typically experience in their daily social interactions. Presumably, many daily social interactions do not involve social rejection and those that do probably involve forms of rejection that are much more subtle and tacit than the overt social exclusion episode that occurred during the Cyberball game. Nonetheless, there were still strong relationships between neural activity to this experimental episode of social rejection and real-world feelings of rejection, indicating that these neural responses still provide a meaningful proxy for real-world social experience.

An additional limitation is that the present study only examined neural activity when social rejection was occurring, but not when individuals were reflecting on past rejection experiences. Thus, we could only assess which neural regions were involved in the possible encoding of these rejection experiences but not which neural regions were involved in the later retrieval of these rejection memories. Future studies would benefit from examining the neural regions associated with reflecting on social experience in addition to the neural regions involved in the initial encoding of these experiences.

In sum, this study utilized a novel technique to assess whether neural reactivity to a single experimental episode of social rejection in the scanner related to real-world social experience. By using daily experience sampling in conjunction with neuroimaging techniques, it is possible to investigate the ways in which neural reactivity to simple tasks relates to or predicts behavior and experience in more naturalistic situations. Examining the relationships between neural responses within the fMRI scanner and real-world experiences may provide important information regarding how individuals experience their social worlds and the neurocognitive processes that underlie these experiences.

\section{References}

Anderson, A. K., \& Phelps, E. A. (2002). Is the human amygdala critical for the subjective experience of emotion? Evidence of intact dispositional affect in patients with amygdala lesions. Journal of Cognitive Neuroscience, 14, 709-720.

Barrett, L. F., \& Barrett, D. J. (2001). An introduction to computerized experience sampling in psychology. Social Science Computer Review, 19, 175-185.

Brewer, J. B., Zhao, Z., Desmond, J. E., Glover, G. H., \& Gabrieli, J. D.
(1998). Making memories: Brain activity that predicts how well visual experience will be remembered. Science, 281, 1185-1187.

Cabeza, R., Prince, S. E., Daselaar, S. M., Greenberg, D. L., Budde, M., Dolcos, F., et al. (2004). Brain activity during episodic retrieval of autobiographical and laboratory events: An fMRI study using a novel photo paradigm. Journal of Cognitive Neuroscience, 16, 1583-1594.

Davis, M., \& Whalen, P. J. (2001). The amygdala: Vigilance and emotion. Molecular Psychiatry, 6, 13-34.

Eisenberger, N. I., Lieberman, M. D., \& Williams, K. D. (2003). Does rejection hurt? An fMRI study of social exclusion. Science, 302, 290 292.

Eysenck, S. B. G., \& Eysenck, H. J. (1975). Manual of the Eysenck Personality Questionnaire. London: Hodder \& Stoughton.

Fenigstein, A., Scheier, M. F., \& Buss, A. H. (1975). Public and private self-consciousness: Assessment and theory. Journal of Consulting and Clinical Psychology, 43, 522-527.

Forman, S. D., Cohen, J. D., Fitzgerald, M., Eddy, W. F., Mintun, M. A., \& Noll, D. C. (1995). Improved assessment of significant activation in functional magnetic resonance imaging (fMRI): Use of a cluster-size threshold. Magnetic Resonance in Medicine, 33, 636-647.

Fredrickson, B. L., \& Kahneman, D. (1993). Duration neglect in retrospective evaluations of affective episodes. Journal of Personality and Social Psychology, 65, 45-55.

Gallagher, H. L., \& Frith, C. D. (2003). Functional imaging of "theory of mind." Trends in Cognitive Sciences, 7, 77-83.

Gusnard, D. A., Akbudak, E., Shulman, G. L., \& Raichle, M. E. (2001). Medial prefrontal cortex and self-referential mental activity: Relation to a default mode of brain function. Proceedings of the National Academy of Sciences, USA, 98, 4259-4264.

Kahneman, D., Fredrickson, B. L., Schreiber, C. A., \& Redelmeier, D. A. (1993). When more pain is preferred to less: Adding a better end. Psychological Science, 4, 401-405.

Lieberman, M. D. (2007). Social cognitive neuroscience: A review of core processes. Annual Review of Psychology, 58, 259-289.

Macrae, C. N., Moran, J. M., Heatherton, T. F., Banfield, J. F., \& Kelley, W. M. (2004). Medial prefrontal activity predicts memory for self. Cerebral Cortex, 14, 647-654.

Mehrabian, A. (1976). Questionnaire measures of affiliative tendency and sensitivity to rejection. Psychological Reports, 38, 199-209.

Morewedge, C. K., Gilbert, D. T., \& Wilson, T. D. (2005). The least likely of times: How remembering the past biases forecasts of the future. Psychological Science, 16, 626-630.

Peyron, R., Laurent, B., \& Garcia-Larrea, L. (2000). Functional imaging of brain responses to pain. A review and meta-analysis. Clinical Neurophysiology, 30, 263-288.

Rainville, P., Duncan, G. H., Price, D. D., Carrier, B., \& Bushnell, M. C. (1997). Pain affect encoded in human anterior cingulate but not somatosensory cortex. Science, 277, 968-971.

Redelmeier, D. A., \& Kahneman, D. (1996). Patients memories of painful medial treatments: Real-time and retrospective evaluations of two minimally invasive procedures. Pain, 66, 3-8.

Reis, H. T., \& Gable, S. L. (2002). Event-sampling and other methods for studying everyday experience (pp. 190-222). In H. T. Reis and C. M. Judd (Eds.), Handbook of Research Methods in Social and Personality Psychology. New York: Cambridge University Press.

Rosenthal, R., \& Rosnow, R. L. (1991). Essentials of behavioral research: Methods and data analysis (2nd ed.). New York: McGraw-Hill.

Updegraff, J. A., Gable, S. L., \& Taylor, S. E. (2004). What makes experiences satisfying? The interaction of approach-avoidance motivations and emotions in well-being. Journal of Personality and Social Psychology, 86, 496-504.

Wager, T. D., Phan, K. L., Liberzon, I., \& Taylor, S. F. (2003). Valence, gender, and lateralization of functional brain anatomy in emotion: A 
meta-analysis of findings from neuroimaging. Neuroimage, 19, 513531.

Wagner, A. D., Schacter, D. L., Rotte, M., Koutstaal, W., Maril, A., Dale, A. M., et al. (1998). Building memories: Remembering and forgetting of verbal experiences as predicted by brain activity. Science, 281, 11881191.

Williams, K. D., Cheung, C. K. T., \& Choi, W. (2000). Cyberostracism:
Effects of being ignored over the Internet. Journal of Personality and Social Psychology, 79, 748-762.

Received August 30, 2006

Revision received February 15, 2007

Accepted February 15, 2007

\section{Correction to Nielsen and Kaszniak (2006)}

In the article "Awareness of Subtle Emotional Feelings: A Comparison of Long-Term Meditators and Nonmeditators," by Lisbeth Nielsen and Alfred W. Kaszniak (Emotion, 2006, Vol. 6, No. 3, pp. 392-405), the copyright attribution is incorrect. The article is in the public domain.

DOI: $10.1037 / 1528-3542.7 .4 .754$ 